\title{
Bayesian inversion of geoelectrical resistivity data
}

\author{
Kim E. Andersen† \\ Aalborg University, Denmark \\ Stephen P. Brooks \\ University of Cambridge, U.K. \\ Martin B. Hansen \\ Aalborg University, Denmark
}

\begin{abstract}
Summary. Enormous quantities of geoelectrical data are produced on a daily basis, and often used for large-scale reservoir modelling. Interpretation of these data requires reliable and efficient inversion methods which adequately incorporate prior information and use realistically complex modelling structures. In this paper we use random coloured polygonal models as a powerful and flexible modelling framework for the layered composition of the Earth and we contrast our approach with earlier methods based upon smooth Gaussian fields. We demonstrate how the reconstruction algorithm may be efficiently implemented through the use of multigrid Metropolis-coupled Markov chain Monte Carlo and illustrate the method on a set of field data.
\end{abstract}

Keywords: Inverse problems; Bayesian inference; Stochastic geometry; Markov chain Monte Carlo; Multigrid methods; Coupling

\section{Introduction}

In electrical impedance tomography (EIT), various current patterns are applied to electrodes on the boundary of an electrically conductive object. The induced surface voltage potentials are measured and used to reconstruct the unknown resistivity distribution within the object. Since electric resistivities in relevant materials are known from experimental studies, it is possible to reconstruct the local composition beneath the surface of the Earth, from measurements made on the surface. Such techniques are widely used in large-scale mapping of groundwater (Christensen and Sørensen, 1998), detection of leaks from landfills, storage ponds and tanks (Daily et al., 1995) and various other geotechnical applications (Panissod et al., 1998). The use of such data to reconstruct details in the Earth requires the solution of an ill-posed inverse problem and it is necessary to restrict the solution space either by regularisation or by utilising prior information.

It is well known that the Earth typically consists of a series of well-defined layers each having its own resistivity. Despite this fact, most commonly-used geoelectrical inversion methods are based upon regularisation techniques that implicitly assume a smooth resistivity distribution and thereby fail to fully describe the true nature of the subterranean structures. An alternative approach which allows for discontinuities in the resistivity profile is based upon the use of coloured polygons. Arak et al. (1993) develop a class of point-based

$\dagger$ Address for correspondence: Kim E. Andersen, Department of Mathematical Sciences, Aalborg University, Fredrik Bajers Vej 7G, DK-9220 Aalborg Ø, Denmark.

E-mail: emil@math.auc.dk 
polygonal models where simple polygons are used to model the structure of interest. These models have been used for restoration of polygonal images by Nicholls (1998), for example. In the following section we develop a subclass of point-based polygonal models in which simple polygons are used as prior model of the layered structure of the Earth.

The search for a solution to an ill-posed inverse problem involves dealing with a possibly highly non-linear relationship between data space and model space in which multiple solutions may be located in separate regions. Problems of this character are, in principle, easily dealt with in the Bayesian framework. Over the past decade the Bayesian approach has experienced an enormous upsurge in interest, both in this and other areas, and powerful new simulation techniques are now available which allow even more realistic and complicated modelling structures. See e.g. Mosegaard and Tarantola (1995), Fox and Nicholls (1997), Mosegaard (1998), Kaipio et al. (2000) and Andersen et al. (2001).

The likelihood function has an open-form expression and its evaluation is highly computer intensive. Moreover, simulation-based posterior inference requires a large number of forward calculations to be performed. Therefore, fast and efficient sampling techniques are required for posterior inference. $\mathrm{Li}$ and Oldenburg (1992) develop a fast technique for predicting experimental geoelectrical data for a given subterranean structure. The technique is based upon a multichannel convolution (MCC) formulation which was refined by Møller et al. (2001). We adopt and develop this formulation to provide a fast and reliable framework for Bayesian inference in this context.

\section{Geoelectrical data acquisition}

Geoelectrical data acquisition systems induce an electrical voltage potential difference $\Delta V$, measured between a pair of potential electrodes $(M, N)$ by injecting a current directly into the Earth using a pair of current electrodes $(A, B)$. See Figure 1(a) for details. Basically, in any electrode configuration $s=(A, B, M, N)$ we obtain a reading $y$ at electrodes $M$ and $N$ from which we can determine a resistivity value for the path of the current from $A-B$ to $M-N$ through the ground at that point. This reading is subject to errors and the true reading should be $\varrho_{s}$ which we call the apparent resistivity. We shall discuss the relationship between $y$ and $\varrho_{s}$ in the next section.

The apparent resistivity $\varrho_{s}$ is measured in $\Omega \cdot \mathrm{m}$ and is obtained by using Ohm's law i.e., when the potential electrodes are close to the current electrodes

$$
\varrho_{s}=g_{s} \Delta V_{s, \rho} / I
$$

where $\rho$ denotes the true subterranean resistivity distribution of the Earth and

$$
g_{s}=2 \pi\left(\frac{1}{\left|x_{A}-x_{M}\right|}-\frac{1}{\left|x_{B}-x_{M}\right|}-\frac{1}{\left|x_{A}-x_{N}\right|}+\frac{1}{\left|x_{B}-x_{N}\right|}\right)^{-1}
$$

is a geometric factor. Here $x_{Q}$ denotes the lateral position of electrode $Q$ for $Q=A, B, M$ and $N$. Obviously, the apparent resistivity depends upon $s$, but also upon the true subterranean resistivity distribution, $\rho$, which describes the resistivity at any point $(x, z)$ under the surface. For the particular case where the Earth is homogeneous $\varrho_{s}$ will, regardless of the location of the four electrodes, be exactly equal to $\rho$. However, the Earth is seldom homogeneous (even at a local level) and instead the apparent resistivity represents an average of the true resistivity distribution of the Earth and formal inversion is needed for further interpretation. 


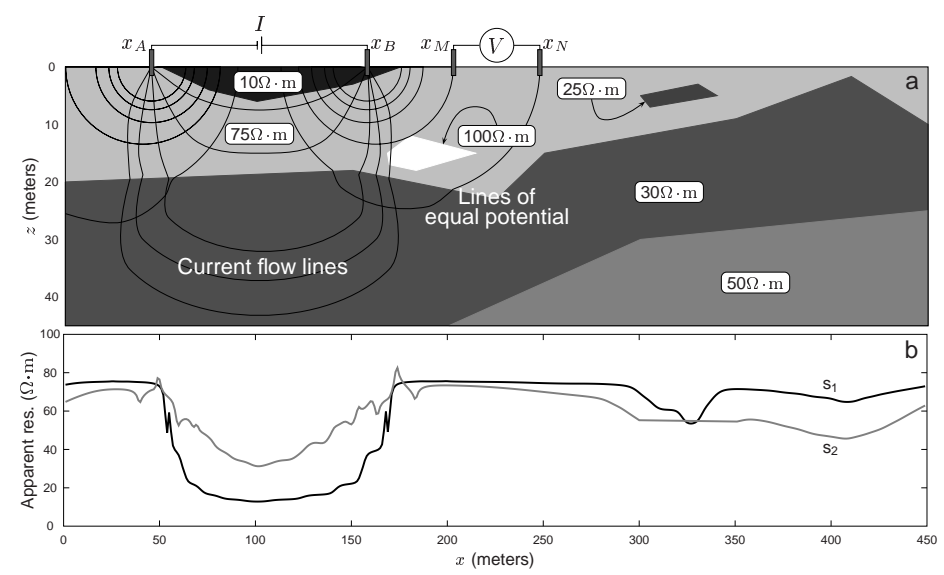

Fig. 1. (a): The apparent resistivity $\varrho_{s}$ can be computed for a given resistivity distribution $\rho$ and electrode configuration $s=(A, B, M, N)$. Here $(A, B)$ denotes the pair of current electrodes and $(M, N)$ denotes the pair of potential electrodes. The voltage potential differences along the surface of the Earth is then recorded by a voltmeter inserted between $M$ and $N$. (b): Theoretical predicted apparent resistivities for two different electrode configurations $s_{1}$ and $s_{2}$.

Clearly, the resulting apparent resistivity data are nonlinear functions of the actual electric resistivities within the Earth together with the electrode configuration. Electrode configurations with large inter-electrode separations are in general better able to recover information deeper from the Earth than electrode configurations with small inter-electrode separation, which are best for shallower depths. Thus, readings are usually taken from a range of configurations in which the distance between electrodes $\mathrm{A}$ and $\mathrm{B}$ remains fixed, but the position of the other two electrodes is varied so as to focus on different depths. For example, Figure 1(b) shows the corresponding apparent resistivities for two different electrode configurations, say $s_{1}$ and $s_{2}$ as they move laterally along the surface. In $s_{2}$ the median depth of investigation (Edwards, 1977) is deeper, resulting in a deeper penetration than in $s_{1}$ i.e., these voltage measurements can be utilised to make deeper reconstructions than $s_{1}$. Notationally, and for any configuration $s$, if we let $\left(x_{s}, z_{s}\right)$ denote the point within the Earth where the median depth of investigation is reached, then $x_{s}$ denotes the lateral focus point and $z_{s}$ the vertical focus point for that configuration. See Edwards (1977) for details.

Data of this sort are often obtained using the pulled array continuous electrical profiling (PACEP) method (Sørensen, 1996; Panissod et al., 1998), a very efficient technique for simultaneous and continuous apparent resistivity data acquisition for many distinct electrode configurations. Electrodes are mounted on a cable towed by a vehicle and measurements are recorded as the cable is drawn across the surface. The PACEP array is based upon one set of current electrodes with fixed inter-electrode distance. For our data, this distance was 30 metres and Figure 2 shows the position of the eight different voltage electrode configurations in the PACEP array, each with its own vertical focus point.

\section{Study area and data}

More than 30 profile kilometres of densely sampled data were collected using the PACEP method in an investigation for smectite-rich clay deposits in the Ølst Formation (HeilmannClausen et al., 1985) in Jutland, Denmark. Upper Palaeocene clay from this formation 


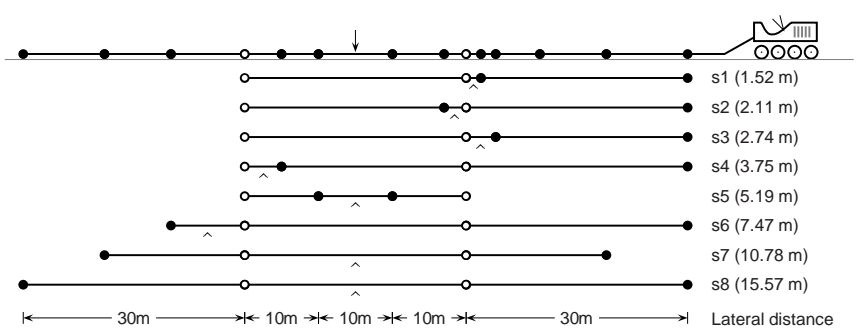

Fig. 2. PACEP array: electrodes are mounted on a cable and towed by a small vehicle. The array has one set of current electrodes (circles) separated by 30 metres. The $\downarrow$ denotes the common lateral reference point, the ${ }^{\wedge}$ denotes the lateral focus of each electrode configuration and quantities in parentheses denote the vertical focus points (figure reproduced with kind permission from Møller et al. (2001)).

outcrops in hills formed by glaciotectonics. These heavily disturbed clay deposits are encompassed by glaciofluvial sand, together with gravel deposits and overlain by a thin clayish till (Nielsen, 1973). Møller et al. (2001) develop a multichannel deconvolution (MCD) for inverting the $\mathrm{MCC}$ formulation in order to reconstruct the resistivity distribution. This MCD technique was applied to segments of about 900 metres of the data. Figure 3(a) depicts a 150 metre long data segment with the corresponding MCD inversion shown in Figure 3(b). Resistivities below $5 \Omega \cdot \mathrm{m}$ are interpreted as Palaeocene clay, whereas resistivities above $100 \Omega \cdot \mathrm{m}$ are interpreted as dry glaciofluvial sand and gravel. Interceding resistivities of approximately $30 \Omega \cdot \mathrm{m}$ are taken to represent a clayish till. The obtained reconstruction reveals a very smooth resistivity distribution. However, geophysicists would expect a somewhat sharper transition between the clay and sand than the one obtained by the MCD technique.
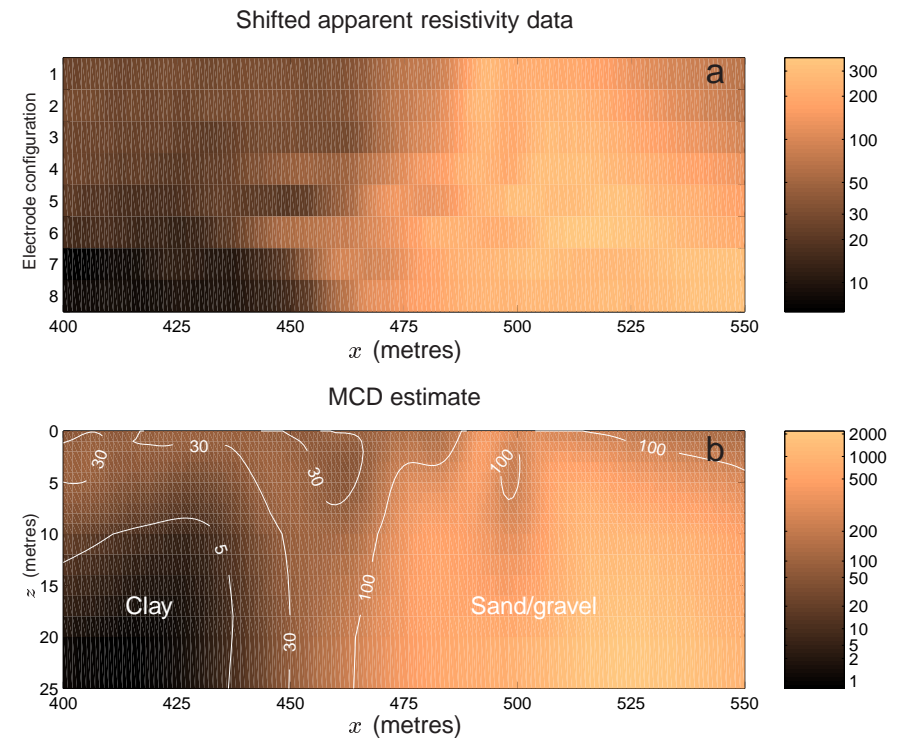

Fig. 3. (a) 1200 apparent resistivity readings, shifted according to their lateral focus points, in a 150 metre long segment from the Ølst Formation, Jutland, Denmark; and (b) the inversion obtained by a MCD along with 5,30 and $100 \Omega \cdot \mathrm{m}$ contour lines describing the believed sedimentary transitions in the MCD reconstruction. 


\section{Outline}

In this paper we revisit the Ølst formation dataset and provide an alternative model which permits the sharp transitions expected by the geophysicists. We begin in Section 2 with a description of the model and prior structures used for the Bayesian analysis. Details of the reconstruction algorithm are given in Section 3 and in Section 4 we use the model for the analysis of Ølst formation field data. Finally, in Section 5, we discuss further potential developments for geoelectrical large-scale mapping. Two appendices provide mathematical details associated with the model and simulation algorithm developed in the paper.

\section{Model}

\subsection{Forward modelling of resistivity data}

Let $S=\left(s_{1}, \ldots, s_{P}\right)$ denote a set of $P$ different electrode configurations. The Earth response $\varrho_{s}\left(x_{s}\right)$ under any electrode configuration $s=(A, B, M, N)$ in $S$ with lateral focus point $x_{s}$ is then given by a transformation $K_{s, \rho}$ depending upon both $s$ and the resistivity distribution $\rho=(\rho(x, z))$ for $(x, z) \in \mathbb{R} \times \mathbb{R}_{+}$. Thus, the apparent resistivity data, measured at the vertical focus point $x_{s}$ on the profile, takes the form

$$
\varrho_{s}\left(x_{s}\right)=K_{s, \rho}\left(x_{s}\right):=g_{s} \Delta V_{s, \rho}\left(x_{s}\right), \quad s=s_{1}, \ldots, s_{P},
$$

where $\Delta V_{s, \rho}\left(x_{s}\right)=V_{s, \rho}\left(x_{N}, 0\right)-V_{s, \rho}\left(x_{M}, 0\right)$ and $V_{s, \rho}$ is the solution to Poisson's equation given by

$$
\nabla \cdot\left(\sigma(x, z) \nabla V_{s, \rho}(x, z)\right)=-I\left[\mathbf{1}\left(x-x_{A}\right)-\mathbf{1}\left(x-x_{B}\right)\right]
$$

subject to the boundary condition $\sigma \partial V_{s, \rho} / \partial \nu=0$ on the surface of $\rho$, where $\sigma=1 / \rho$ is the conductivity and $I$ denotes the current flowing into the ground at $x_{A}$ and out at $x_{B}$. Here the $z$-axis points downwards and the $x$-axis is the horizontal axis along which the cable is drawn.

Logarithmic transformation of both the data and the resistivity distribution ensures nonnegative resistivities. Therefore, we let $\rho^{\delta}=\log \rho-\log \rho_{\text {ref }}$ denote the logarithmic resistivity perturbation and let $\varrho_{s}^{\delta}=\log \varrho_{s}-\log K_{s}\left(\rho_{\text {ref }}\right)$ denote the logarithmic data perturbation, where $\rho_{\text {ref }}$ is a reference resistivity distribution. Expanding (1) in a first order Taylor series with respect to $\rho_{\text {ref }}$ gives the Born approximation (Boerner and West, 1989). Furthermore, if we let $\rho_{\text {ref }}$ denote a homogeneous half-space, which is translationally invariant along the profile, then the Fréchet derivative $\Phi_{s}$ inherits translational invariance from $\rho_{\text {ref. }}$ Subsequently the modified Born approximation is a convolution between $\Phi_{s, \rho_{\mathrm{ref}}}(0, z, \cdot)$ and $\rho^{\delta}(\cdot, z)$ i.e.,

$$
\varrho_{s}^{\delta}\left(x_{s}\right) \simeq \int_{0}^{\infty} \int_{-\infty}^{\infty} \Phi_{s, \rho_{\mathrm{ref}}}\left(0, z, x_{s}-x\right) \rho^{\delta}(x, z) d x d z,
$$

where the 2-dimensional form of the Fréchet derivative for $\varrho_{s}$ with respect to $\rho$ is

$$
\Phi_{s, \rho}=\frac{g_{s}}{I} \frac{\partial V_{s, \rho}\left(x_{s}, z_{s}\right)}{\partial \rho(x, z)} .
$$

Closed form expressions for the Fréchet derivative evaluated for a translationally invariant half-space along the profile are available; derivations can be found in Loke and Barker (1995). However, for clarity we briefly state the closed form expressions in Appendix A.

The resistivity distribution is discretised in the $z$-direction into $m$ layers i.e., $\rho(x, z)$ is approximated by a function $\rho(x, l)$ for layers $l=1, \ldots, m$, which describes the lateral 
variation in a layer. We therefore define the vertically averaged Fréchet derivative in layer $l$ by

$$
\Phi_{s, \rho_{\mathrm{ref}}}^{*}(x, l)=\int_{z_{l-1}}^{z_{l}} \Phi_{s, \rho_{\mathrm{ref}}}(0, z, x) d z .
$$

If the data are sampled regularly in all electrode configurations in $S$ along the profile, then a multichannel convolution formulation is obtained i.e.,

$$
\varrho_{s}^{\delta}\left(x_{s}\right) \simeq \sum_{l=1}^{m} \int_{-\infty}^{\infty} \Phi_{s, \rho_{\mathrm{ref}}}^{*}\left(x_{s}-x, l\right) \rho^{\delta}(x, l) d x
$$

which, by Fourier transformation, gives the sum of products

$$
\widetilde{\varrho}_{s}^{\delta}(k) \simeq \sum_{l=1}^{m} \widetilde{\Phi}_{s, \rho_{\mathrm{ref}}}^{*}(k, l) \widetilde{\rho}^{\delta}(k, l) .
$$

Here, the tilde denotes Fourier domain variables and the wavenumber $k=1 / \lambda$ denotes the reciprocal wavelength. Hence, for each wavenumber, equation (3) yields a linear system of $P$ equations with $m$ unknowns i.e., the large 2-dimensional spatial problem has decoupled into many small 1-dimensional problems in the wave number domain. If we assume that $M$ samples have been recorded in $P$ different electrode settings then we must solve an $M P$ by- $M P$ problem in the space domain, whereas the decoupled problem in the wave number domain reduces to $M$ small $P$-by- $P$ problems. The MCD technique presented in Møller, Jacobsen and Christensen (2001) solves this linear system. We note that the number of readings appears in the Fréchet derivative, whereas the coarseness affects only $\rho$. This observation will be used in Section 3.

Implementation of (3) leads to the use of a discrete Fourier transform and thereby a discretisation in the horizontal direction. Consequently, theoretical apparent resistivity data in the space domain is obtained by inverse Fourier transformation of $\widetilde{\varrho}_{s}^{\delta}(k)$. The multichannel convolution formulation derived here avoids the evaluation of the earth response $K_{s, \rho_{\text {ref }}}$ in (1) as a layered reference medium $\rho_{\text {ref }}$ is imposed. However, evaluation of the Fréchet derivative requires a deeper understanding of the operator $K_{s, \rho}$.

\subsection{Deriving the likelihood function}

The random noise process imposed on the apparent resistivity data has, by its very nature, a highly complicated structure. The likelihood function should be derived under the assumption that the random errors can be split into three terms: one term $\eta_{S}$ constant for all $x_{s}$, describing the spatial small-scale variation of the electrical resistivities in the Earth; another term, $\eta_{L}\left(x_{s}\right)$, describing the linearisation error induced by the Born approximation under electrode configuration $s$ at $x_{s}$; and finally a third term, $\eta_{N}\left(x_{s}\right)$, describing the noise inherent in the instrumentation - essentially measurement error due to e.g. electrode polarisation and telluric currents at $x_{s}$. An observational model using first order approximations is then obtained by setting

$$
y\left(x_{s}\right)=\log K_{s, \rho_{\text {ref }}}\left(x_{s}\right)+\log K_{s, \eta_{S}}\left(x_{s}\right)+\eta_{L}\left(x_{s}\right)+\eta_{N}\left(x_{s}\right) .
$$

It is well known that the resistivity, like other petrophysical parameters, oscillates 'slowly' with amplitudes of typically 1 to 10 per cent over spatial distance (Jacobsen, 1993). 
Moreover, synthetic data based upon an exact, but highly computer intensive finite difference formulation (McGillivray, 1992) can be compared to the linear Born approximation response we have adopted. The full non-linear response for low-contrast models show surprisingly concordance with the full non-linear response. However, the responses for the high-contrast model reveals a significant non-linearity error with a long-term correlation, which has to be taken into consideration when specifying the random error process. Finally, commonly-used geoelectrical data acquisition systems are known to be very accurate, though observation errors are likely to increase with increasing electrode separation. Moreover, for pulled arrays some cross-correlation may be expected, as distinct electrode configurations may share some of the moving electrodes.

Geophysicists conveniently model the observation error by a white noise process. However, field data appear to exhibit signs of a smooth correlation structure. Thus, we make the more realistic assumption that we observe data $y\left(x_{s}\right)=\log \varrho_{s}\left(x_{s}\right)+\epsilon\left(x_{s}\right)$, where $\epsilon\left(x_{s}\right)$ denotes a stationary Gaussian process with zero mean, $\mathbb{E} \epsilon\left(x_{s}\right)=0$, and a covariance structure from the Matérn family (Matérn, 1986) with covariance function

$$
\operatorname{Cov}(d)=\sigma^{2} \frac{1}{2^{\nu-1} \Gamma(\nu)}(d / L)^{\nu} \mathcal{K}_{\nu}(d / L), \quad \nu>0,
$$

where $d$ denotes the spatial distance between focus points, $L>0$ is the correlation length and $\mathcal{K}_{\nu}$ is the modified Bessel function of the second kind of order $\nu$. The likelihood function can now be derived under the assumption that the errors are distributed as Gaussian with mean zero and covariance matrix $\Sigma=\Sigma_{\nu, L, \sigma}$ described by (4) i.e.,

$$
p(y \mid \rho, \Sigma) \propto|\Sigma|^{-1 / 2} \exp (-G(\rho ; \Sigma))
$$

with

$$
G(\rho ; \Sigma)=\left(y-\log K_{S}(\rho)\right)^{\top} \Sigma^{-1}\left(y-\log K_{S}(\rho)\right),
$$

where $y=\left(y\left(x_{s_{1}}^{1}\right), \ldots, y\left(x_{s_{1}}^{N}\right), \ldots, y\left(x_{s_{P}}^{1}\right), \ldots, y\left(x_{s_{P}}^{N}\right)\right)^{\top}$ and

$$
K_{S}(\rho)=\left(K_{s_{1}, \rho}\left(x_{s_{1}}^{1}\right), \ldots, K_{s_{1}, \rho}\left(x_{s_{1}}^{N}\right), \ldots, K_{s_{P}, \rho}\left(x_{s_{P}}^{1}\right), \ldots, K_{s_{P}, \rho}\left(x_{s_{P}}^{N}\right)\right)^{\top}
$$

is the earth response obtained when evaluating $K_{s, \rho}$ for all electrode configurations in $S$. Here $N$ denotes the number of sampling locations for each electrode configuration.

\subsection{Specification of the resistivity distribution}

Let $\Omega \subseteq \mathbb{R}^{2}$ denote the interior of a rectangular polygon with corners $h=\left\{h_{1}, h_{2}, h_{3}, h_{4}\right\}$. The set of all finite sets of vertices in $\Omega$ is denoted by $X_{\Omega}$ and the set of all finite sets of vertices on the boundary is denoted by $X_{\partial \Omega}$. If we let $X_{\Omega}^{(m)}$ denote the set of all vertex sets comprising $m \geq 0$ vertices in $\Omega$ then $X_{\Omega}=\bigcup_{m=0}^{\infty} X_{\Omega}^{(m)}$. Similarly, if we let $X_{\partial \Omega}^{(n)}$ denote the set of vertices on $\partial \Omega$ comprising $n \geq 0$ vertices then $X_{\partial \Omega}=\bigcup_{n=0}^{\infty} X_{\partial \Omega}^{(n)}$.

The embedding of a finite graph in a bounded region of the plane is defined as a planar straight line graph (Brown, 1964) if it has no coincident vertices and no intersecting edges. The degree of a vertex in a graph is defined as the number of incident edges. By $V$-vertices we refer to any vertices of degree 2 and those $V$-vertices whose edges are perpendicular, we refer to as $L$-vertices. Finally, vertices with degree 3 are defined as $T$-vertices if two of the incident edges are parallel. See Figure 4 for details. 


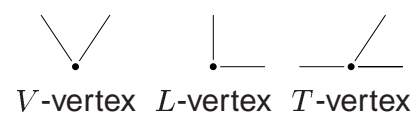

Fig. 4. The $V$-, $L$ - and $T$-vertices used in the polygonal model.

Let $v \in X_{\Omega}$ and $l \in X_{\partial \Omega}$ and consider the subset $\Psi_{\Omega}(v, l, h)$ of planar straight line graphs with vertices $v \cup l \cup h$ for which: (1) the vertices in $v$ are $V$-vertices; (2) the vertices in $l$ are $T$-vertices; and (3) the vertices in $h$ form the domain $\Omega$ by $L$-vertices. The set $\Psi_{\Omega}(v, l, h)$ is referred to as the set of admissible polygonal models which can be constructed in $\Omega$ with vertex set $(v, l, h)$. See Figure 5 for two examples with the same vertex set. The space of admissible polygonal models in $\Omega, \Psi_{\Omega}$, is defined as the union over all polygonal models in $\Omega$ :

$$
\Psi_{\Omega} \equiv \bigcup_{(v, l) \in X_{\Omega} \times X_{\partial \Omega}} \Psi_{\Omega}(v, l, h) .
$$

Note that the definition of admissible polygonal models allows the graphs in $\Psi_{\Omega}(v, l, h)$ to be disconnected i.e., there may be self-contained masses within $\Omega$ that cannot be reached from the boundary by following any path in the graph.

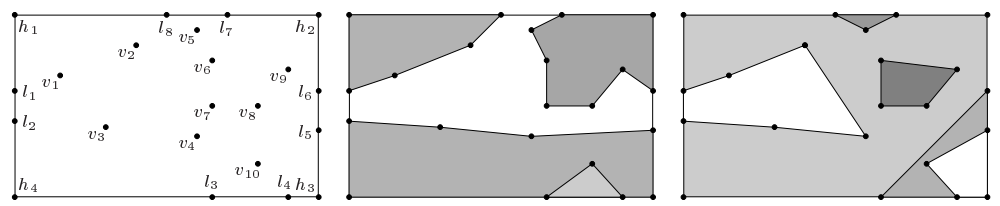

Fig. 5. For fixed vertex sets $v$ and $l$ we may obtain many distinct realisations from the basic random polygonal model distribution. Note how irregular masses (as opposed to layers) may be constructed by joining interior vertices only. The polygonal faces are shown with different shadings.

A random polygonal model is a measurable map $\psi$ from some measure space to $\left(\Psi_{\Omega}, \mathcal{B}_{\Omega}\right)$, where $\mathcal{B}_{\Omega}$ is a $\sigma$-algebra of subsets of $\Psi_{\Omega}$. In the present paper we use the construction by Arak and Surgailis $\left(1989\right.$, p. 547) for $\mathcal{B}_{\Omega}$. Let $A \in \mathcal{B}_{\Omega}$, then a base measure $\pi$ on the set of polygonal models is defined by

$$
\pi(A)=\sum_{n=0}^{\infty} \sum_{m=0}^{\infty} \int_{X_{\Omega}^{(m)}} d v_{1} d v_{2} \cdots d v_{m} \int_{X_{\partial \Omega}^{(n)}} d l_{1} d l_{2} \cdots d l_{n} \sum_{\psi \in \Psi_{\Omega}(v, l, h)} \mathbf{1}(\psi \in A) .
$$

Now, let $\lambda_{\Omega}$ and $\lambda_{\partial \Omega}$ denote the intensities of two independent Poisson point processes in $\Omega$ and on $\partial \Omega$ respectively. The distribution of $\psi$ is then defined by the density

$$
p(\psi)=\frac{1}{\mathcal{K}_{\Omega}} \lambda_{\Omega}^{m(v)} \lambda_{\partial \Omega}^{n(l)},
$$

which is absolutely continuous with respect to $\pi$. We note that the number of polygonal models in $\Psi_{\Omega}(v, l, h)$ is bounded by the number of triangulations on the same vertex set. Consequently the normalising constant $\mathcal{K}_{\Omega}$ is finite whenever $\Omega$ has non-zero area (Nicholls, 1998, Theorem 1).

The polygonal models in $\Psi_{\Omega}$ are used to segment the study area $\Omega$ into regions of uniform resistivities. Each region is referred to as a polygonal face, which takes a certain 
colour corresponding to its resistivity. We assume that $\psi$ is a fixed polygonal model and let $f_{\psi}$ denote the number of distinct faces in $\psi$. For simplicity we also assume that the set $\mathcal{J}$ of allowable face colours is finite and that $c_{i}$ denotes the colour of the $i$ th face in $\psi$ for $i=1,2, \ldots, f_{\psi}$. Then, the set of all possible face colourings of $\psi$ is given by $C(\psi)=\mathcal{J}^{f_{\psi}}$. Finally, if we let $\rho_{\psi, c}: \Omega \rightarrow \mathcal{J}$ define the colouring map from each point $(x, z)$ in $\Omega$ to the corresponding colour in $\mathcal{J}$, then the set $\boldsymbol{\Psi}_{\Omega, C}$ of all colouring maps which can be constructed from random polygonal models in $\Omega$ is given by

$$
\Psi_{\Omega, C} \equiv \bigcup_{\psi \in \Psi_{\Omega}} \bigcup_{c \in C(\psi)}\left(\rho_{\psi, c}(x, z)\right)_{(x, z) \in \Omega} .
$$

We define a prior distribution on $\Psi_{\Omega, C}$ with respect to the product of $\pi$ and the counting measure by

$$
p_{\Omega, C}(\rho)=\frac{1}{\mathcal{K}_{\Omega}} \frac{1}{|C(\psi)|} \lambda_{\Omega}^{m(v)} \lambda_{\partial \Omega}^{n(l)} \exp \left\{-\theta_{\zeta} \zeta(\rho)\right\} \mathbf{1}[c \in C(\psi)]
$$

where, for notational convenience, we write $\rho=\rho_{\psi, c}$ and $\zeta(\rho)$ denotes the overall length of the discontinuity set of $\rho$ i.e., the summed length of all edges between polygonal faces of different colour in $\rho$. The density is obviously well-defined and in Section 3 we show how the intractable normalisation constant $\mathcal{K}_{\Omega}$ can be treated via Markov chain Monte Carlo (MCMC) simulation (see e.g. Brooks, 1998; Robert and Casella, 1999).

The positive constant $\theta_{\zeta}$ is a tuning parameter, used to control the number of distinct coloured polygonal regions in the reconstruction. Note that by choosing a large value for $\theta_{\zeta}$, we get a reasonably flat prior on $\rho_{\psi, c}$ and that the boundary $\partial \Omega$ does not contribute to $\zeta(\rho)$. This corresponds to a free boundary condition on the model (Nicholls, 1998). For parsimony, we use a single intensity $\lambda$ by setting $\lambda_{\Omega}=\lambda^{2}$ and $\lambda_{\partial \Omega}=\lambda$. Obviously, the average number of polygons rises with $\lambda$, whereas the number of distinct coloured polygons decreases as $\theta_{\zeta}$ increases. Consequently the appearance of realisations from the prior distribution is controlled solely by $\lambda$ and $\theta_{\zeta}$. Figure 6 shows eight simulated realisations under the prior on the unit square for different values of $\lambda$ and $\theta_{\zeta}$ with the number of allowable face colours, $|\mathcal{J}|=5$. Note how the number of distinct coloured areas increases as $\theta_{\zeta}$ decreases.

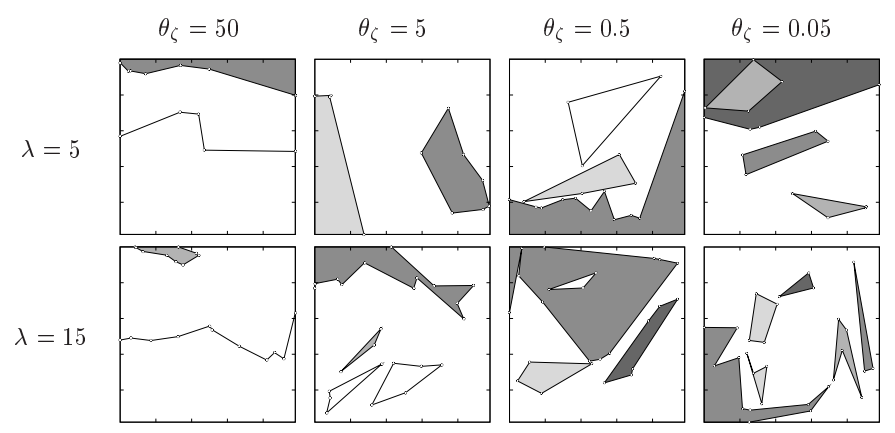

Fig. 6. Samples from the prior distribution on the unit square $\left(\lambda_{\Omega}=\lambda^{2}, \lambda_{\partial \Omega}=\lambda\right.$ and $\left.|\mathcal{J}|=5\right)$.

We will assume a priori that all three parameters in the covariance structure defined in Equation (4) are independently gamma distributed i.e.,

$$
\sigma^{-2} \sim \Gamma\left(a_{\sigma}, b_{\sigma}\right), \quad \nu \sim \Gamma\left(a_{\nu}, b_{\nu}\right) \quad \text { and } \quad L \sim \Gamma\left(a_{L}, b_{L}\right) .
$$


The stochastic quantities in the model and their interdependent relations can be summarised by the directed acyclic graph (DAG; Pearl, 1988) in Figure 7.

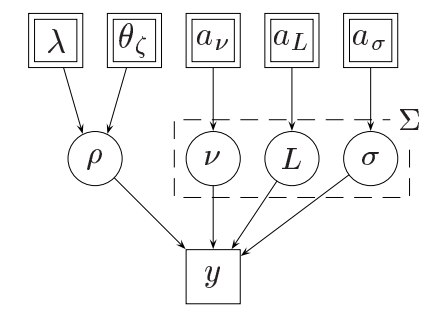

Fig. 7. DAG representing the model used in the geoelectrical reconstruction algorithm. The single edged square denotes the observed data $y$; the single edged circles denote the latent variables; and the double edged boxes represent observed, constant parameters.

\section{Geoelectrical data analysis with coloured polygonal models}

Inference on the composition of the Earth is based upon the posterior distribution

$$
p_{\Omega, C}\left(\rho_{\psi, c}, \nu, L, \sigma \mid y\right) \propto p_{\Omega, C}\left(\rho_{\psi, c}\right) p\left(y \mid \rho_{\psi, c}, \nu, L, \sigma\right) p(\nu) p(L) p(\sigma),
$$

where $p(\nu), p(L)$ and $p(\sigma)$ denote densities of the prior distributions for the variance parameters. This posterior distribution may be summarised by statistics of interest such as the maximum a posteriori estimate

$$
\underset{\substack{\rho_{\psi, c} \in \Psi_{\Omega, C} \\ \nu, L, \sigma \in \mathbb{R}^{-}}}{\arg \max } p_{\Omega, C}\left(\rho_{\psi, c}, \nu, L, \sigma \mid y\right)
$$

or the posterior mean of every pixel value in $\rho_{\psi, c}$

$$
\iint_{\Psi_{\Omega, C}} \iiint_{\mathbb{R}_{+}^{3}} \rho_{\psi, c}(x, z) p_{\Omega, C}\left(\rho_{\psi, c}, \nu, L, \sigma \mid y\right) d \rho_{\psi, c} d \nu d L d \sigma .
$$

Explicit evaluation is impossible but MCMC methods provide an alternative technique based upon samples from the posterior distribution. Posterior means may be estimated by the corresponding sample mean, for example. These draws from the posterior are obtained via MCMC which simulates a Markov chain $\left\{\rho_{t}\right\}$ with a pre-specified invariant distribution. Essentially, MCMC methods work by taking some initial configuration and proposing changes to it which are either accepted or rejected. In the context of our example, sensible changes might include translating the vertices in $(v, l)$, adding or deleting vertices, and changing the colouring. In order to investigate the state space efficiently, we suggest using three within-model moves i.e., those which retain the current set of points in the resistivity model $\rho_{\psi, c}$, and three between-model moves that insert and delete polygons/vertices in the current model. It is obvious that the insertion, deletion and recolouring moves are the minimum requirement for investigating the state space completely. However, the translation move that allows for the movement of the polygons supplements these to make movement around the state space more rapid. The six different proposal types must be scaled appropriately to make them as efficient as possible and optimal scales are often found via pilot tuning. See Figure 8 for details. 

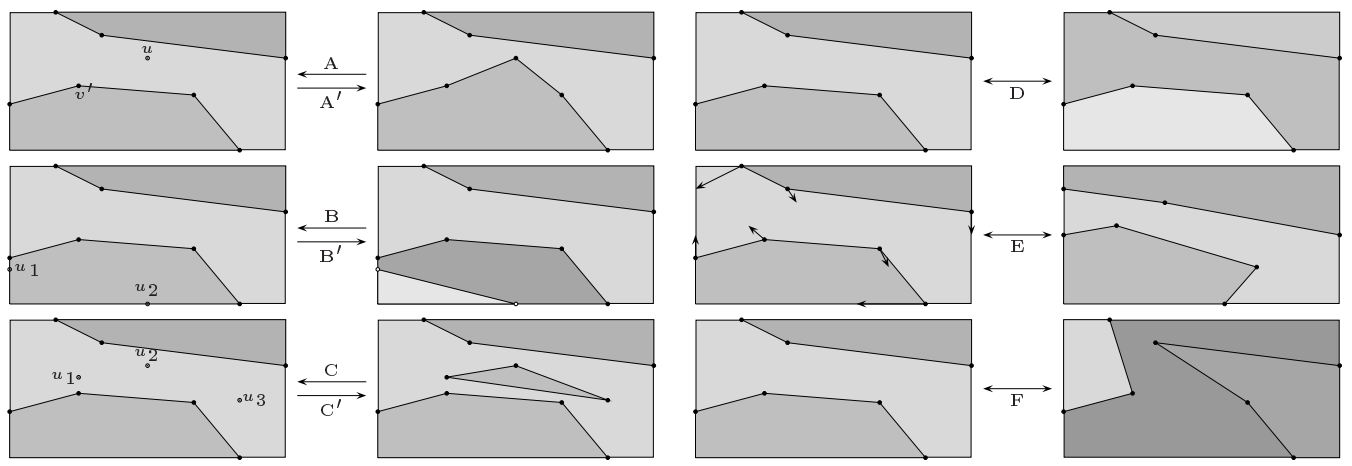

Fig. 8. The proposed updates used in the MCMC reconstruction algorithm. Shown are the different possible transitions for adding $(A)$ and deleting $\left(A^{\prime}\right)$ vertices within polygons, adding $(B)$ and deleting $\left(B^{\prime}\right)$ boundary polygons, adding $(C)$ and deleting $\left(C^{\prime}\right)$ interior polygons, recolouring polygonal faces $(D)$, translating vertices in polygons $(E)$ and shifting edges $(F)$.

Within each update, we begin by deciding which particular move we will attempt, according to specified probabilities $\tau_{\mathrm{A}}, \tau_{\mathrm{A}^{\prime}}, \ldots, \tau_{\mathrm{F}}$. Move-type $\mathrm{A}$ involves the introduction of a new vertex in a polygon i.e., the vertex set $v$ is changed. The reverse move in which a vertex is deleted is referred to as move-type $\mathrm{A}^{\prime}$. Move-types $\mathrm{B}$ and $\mathrm{C}$ involve the introduction of a new polygonal face, whereas the reverse move-types $\mathrm{B}^{\prime}$ and $\mathrm{C}^{\prime}$ enable us to delete polygons ( $v$ and $l$ are changed). Move-type $\mathrm{D}$ alters the resistivity assigned to the polygonal faces $(c$ is changed) and move-type E translates the vertices in $\psi$ in a given direction ( $v$ and $l$ are changed). Finally, move-type F flips two edges. Obviously, move types A and E only make changes to the polygonal model $\psi$, whereas move type $\mathrm{D}$ only updates the colouring of $\psi$. The remaining move types $(\mathrm{B}, \mathrm{C}$ and $\mathrm{F}$ ) make changes to both $\psi$ and $c$. Note also that these moves are reversible by design.

We shall distinguish between three separate simulation algorithms that we later combine to construct a simulation technique suitable for the geoelectrical reconstruction problem: (1) the Metropolis-Hastings updating scheme (Metropolis et al., 1953; Hastings, 1970) for within model moves i.e., move-types D, E and F; (2) the reversible jump MCMC update (Green, 1995), which allows us to perform between-model moves (move-types A, B and C); and (3) finally we modify the Metropolis-coupled MCMC (MCMCMC) method (Geyer, 1991) as a means of increasing the computational efficiency of the overall process.

\subsection{Metropolis-Hastings updates}

The Metropolis-Hastings prescription is used to construct a transition kernel which updates the model parameters whilst retaining the stationary distribution of interest i.e., the posterior distribution given in (7). Given that the chain is in state $\rho_{\psi, c}$ for a given $x, v, \psi$ and $c$ at time $t$, a proposed new state $\rho_{\psi^{\prime}, c^{\prime}}$ is drawn from some (essentially arbitrary) proposal kernel $q\left(\rho_{\psi, c} ; \rho_{\psi^{\prime}, c^{\prime}}\right)$ and then subsequently accepted with probability

$$
\alpha\left(\rho_{\psi, c} ; \rho_{\psi^{\prime}, c^{\prime}}\right)=\min \left\{1, \frac{p_{\Omega, C}\left(\rho_{\psi^{\prime}, c^{\prime}}, \nu, L, \sigma \mid y\right) q\left(\rho_{\psi^{\prime}, c^{\prime}} ; \rho_{\psi, c}\right)}{p_{\Omega, C}\left(\rho_{\psi, c}, \nu, L, \sigma \mid y\right) q\left(\rho_{\psi, c} ; \rho_{\rho^{\prime}, c^{\prime}}\right)}\right\}
$$

and $\rho_{t+1}=\rho_{\psi^{\prime}, c^{\prime}}$. If the proposal is rejected, then $\rho_{t+1}=\rho_{\psi, c}$. Note, the dependence of $\psi(v, l)$ on the vertices $(v, l)$ is suppressed here. 
For illustration, we give explicit descriptions and calculations for translating vertices in $v$ (move-type $\mathrm{E}$ ) below. Details of the remaining within-model move-types are given in Appendix B. Move-type $\mathrm{E}$ is chosen with probability $\tau_{\mathrm{E}}$. This move is performed by considering in turn the vertices in $(v, l)$ and updating each vertex by selecting a new vertex uniformly within some small region around the current vertex. More specifically, let $p=(x, z)$ denote any vertex in $(v, l)$. If $p \in v$, then we propose $p^{\prime}=(x+d x, z+d z)$, where $d x$ and $d z$ are uniformly distributed on the intervals $(-a, a)$ and $(-b, b)$, respectively. However, if $p \in l$, then we propose sliding $p$ counter-clockwise along the boundary for some distance drawn from a uniform distribution on the interval $(-l, l)$. See Figure 9 for details.

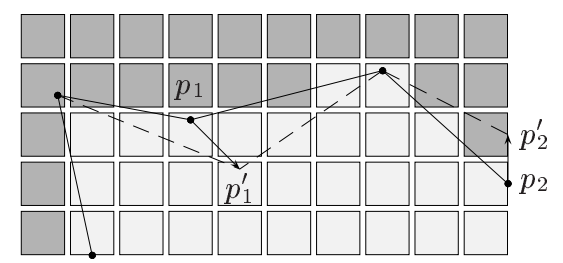

Fig. 9. Any update of $\psi$ or $c$ requires a new approximating profile to be constructed. Here the updating process is illustrated for translating vertices in $(v, l)$. When updating an interior vertex $p$, a new candidate is drawn uniformly from a box centered in $p$. Boundary vertices are updated in a similar way. Note how each pixel within $\rho$ is assigned its most dominating colour.

In proposing a change to any of these two different types of vertices, we propose a move from $(v, l)$ to $\left(v^{\prime}, l\right)$, or from $(v, l)$ to $\left(v, l^{\prime}\right)$, from which $\psi^{\prime}$ is obtained. Hence we propose a move $\rho_{\psi, c}$ to $\rho_{\psi^{\prime}, c}$, with $q_{\mathrm{E}}\left(\rho, \rho_{\psi^{\prime}, c}\right)=1 /(4 a b)$ for an interior vertex and $q_{\mathrm{E}}\left(\rho, \rho_{\psi^{\prime}, c}\right)=$ $1 /(2 l)$ for a boundary vertex. Thus $q_{\mathrm{E}}$ is symmetric and the posterior ratio in (8), with $p_{\Omega, C}(\rho, \nu, L, \sigma \mid y)$ defined in (7), is given by

$$
\frac{p_{\Omega, C}\left(\rho_{\psi^{\prime}, c}, \nu, L, \sigma \mid y\right)}{p_{\Omega, C}\left(\rho_{\psi, c}, \nu, L, \sigma \mid y\right)}=\frac{|C(\psi)| \lambda_{\Omega}^{n} \lambda_{\partial \Omega}^{m} \exp \left(-\theta_{\zeta} \zeta\left(\rho_{\psi^{\prime}, c}\right)-G\left(\rho_{\psi^{\prime}, c} ; \Sigma\right)\right)}{\left|C\left(\psi^{\prime}\right)\right| \lambda_{\Omega}^{n} \lambda_{\partial \Omega}^{m} \exp \left(-\theta_{\zeta} \zeta\left(\rho_{\psi, c}\right)-G\left(\rho_{\psi, c} ; \Sigma\right)\right)} .
$$

Note that the prior densities cancel and the colouring of the polygonal model is unchanged. The acceptance probability is simply

$$
\alpha_{\mathrm{E}}\left(\rho_{\psi, c} ; \rho_{\psi^{\prime}, c}\right)=\min \left\{1, \exp \left[\theta_{\zeta}\left(\zeta\left(\rho_{\psi, c}\right)-\zeta\left(\rho_{\psi^{\prime}, c}\right)\right)+G\left(\rho_{\psi, c} ; \Sigma\right)-G\left(\rho_{\psi^{\prime}, c} ; \Sigma\right)\right] .\right.
$$

Updating of the variance parameters $\nu, L$ and $\sigma$ also require Metropolis-Hastings updates. Let $\rho=\rho_{\psi, c}$ and $\Sigma_{\nu, L, \sigma}=\sigma^{2} \Sigma_{\nu, L}$, then at every iteration of the chain we update the precision $\tau \equiv \sigma^{-2}$ by choosing as proposal distribution

$$
\pi\left(\tau \mid \rho, \Sigma_{\nu, L}\right) \propto \tau^{a_{\sigma}+N P / 2-1} \exp \left[-\tau^{-1}\left(b_{\sigma}+\left(\log K_{S}(\rho)-y\right)^{\top} \Sigma_{\nu, L}^{-1}\left(\log K_{S}(\rho)-y\right)\right) / 2\right],
$$

i.e., the proposal is a gamma distribution with parameters $a_{\sigma}+N P / 2$ and $b_{\sigma}+(y-$ $\left.\log K_{S}(\rho)\right)^{\top} \Sigma_{\nu, L}^{-1}\left(y-\log K_{S}(\rho)\right) / 2$, where $N$ denotes the number of sampling locations on the profile surface and $P$ denotes the number of different electrode configurations in $S$. It is easily shown that if this distribution is chosen as the proposal, then the corresponding acceptance probability is identically equal to one and this update is known as a Gibbs sampler update - a special form of Metropolis-Hastings. The remaining variance parameters $\nu$ and $L$ also require updating and, for efficiency, this is done once every 20 iterations. 
Because of the complicated form of $\Sigma_{\nu, L}$ we update these two parameters using the more general Metropolis-Hastings update. Suppose that we attempt an update of the correlation length $L$, and that a new candidate $L^{\prime}$ is drawn from a gamma distribution with parameters $a_{L}$ and $b_{L}$. Then the acceptance probability takes the form

$$
\begin{aligned}
\alpha\left(L ; L^{\prime}\right)=\min \{1, & \left|\Sigma_{\nu, L^{\prime}}^{-1} \Sigma_{\nu, L}\right|^{1 / 2}\left(\frac{L^{\prime}}{L}\right)^{a_{L}-1} \\
& \left.\times \exp \left[\frac{\tau}{2}\left(\log K_{S}(\rho)-y\right)^{\top}\left(\Sigma_{\nu, L}^{-1}-\Sigma_{\nu, L^{\prime}}^{-1}\right)\left(\log K_{S}(\rho)-y\right)+b_{L}\left(L-L^{\prime}\right)\right]\right\} .
\end{aligned}
$$

The acceptance probability for updating the smoothness parameter $\nu$ is obtained in a similar way.

These Metropolis-Hastings updates are used to update the state vector essentially moving the current polygon configuration. In order to move between configurations with different numbers of vertices, we require what are known as reversible jump MCMC (RJMCMC) updates, since such updates involve moving between states of different dimensions. For example, the introduction of a new coloured polygonal face to the current coloured configuration will increase the dimensionality of the state vector, since additional parameters will be needed to describe the new configuration.

\subsection{Reversible jump Markov chain Monte Carlo}

Suppose a dimension-changing move $m$ is proposed and the proposal $\rho_{\psi^{\prime}, c^{\prime}}$ is generated by a deterministic invertible function $f\left(\rho_{\psi, c}, u\right)$, where $u$ is a continuous random variable. Then Green (1995) shows, that if $r_{m}\left(\rho_{\psi, c}\right)$ denotes the probability of choosing move type $m$ when in state $\rho_{\psi, c} ; q(u)$ denotes the density function of $u$, and $p_{\Omega, C}\left(\rho_{\psi, c}, \nu, L, \sigma \mid y\right)$ denotes the posterior density of $\rho_{\psi, c}$, then the corresponding acceptance probability becomes

$$
\alpha\left(\rho_{\psi, c} ; \rho_{\psi^{\prime}, c^{\prime}}\right)=\min \left\{1, \frac{p_{\Omega, C}\left(\rho_{\psi^{\prime}, c^{\prime}}, \nu, L, \sigma \mid y\right) r_{m^{\prime}}\left(\rho_{\psi^{\prime}, c^{\prime}}\right)}{p_{\Omega, C}\left(\rho_{\psi, c}, \nu, L, \sigma \mid y\right) r_{m}\left(\rho_{\psi, c}\right) q(u)}\left|\frac{\partial f\left(\rho_{\psi, c}, u\right)}{\partial\left(\rho_{\psi, c}, u\right)}\right|\right\},
$$

where $m^{\prime}$ denotes the reverse move to $m$. We note that the final term in the above ratio is the Jacobian arising from the change of variables associated with moving from one space to the other. Algorithmically, the reversible jump updating procedure proceeds identically to that for Metropolis-Hastings updates.

For illustration, we give explicit descriptions and calculations of move-types $\mathrm{A}$ and $\mathrm{A}^{\prime}$ and their corresponding acceptance probabilities below. Details of the remaining dimension changing move-types are also given in Appendix B. Assume that configuration $\rho_{\psi, c}$ consists of $n$ interior vertices and $m$ boundary vertices then for a birth, a new vertex is proposed by sampling a vertex $u=(x, z)$ uniformly in $\Omega$ i.e., $u$ has density $s(u)=1 / A(\Omega)$. Next, select a vertex $v$ uniformly amongst the $n$ existing interior vertices in $\psi$ and add $u$ between the vertex $v$ and one of its neighbours, say $v^{\prime}$. The original edge $\left(v, v^{\prime}\right)$ in $\psi$ is then replaced in $\psi^{\prime}$ by $\left(v, u, v^{\prime}\right)$ which is automatically rejected if either one of these edges crosses any other in $\psi^{\prime}$ i.e., $\psi^{\prime} \notin \mathbf{\Psi}_{\Omega}$. The proposal probability for this move is then

$$
q_{\mathrm{A}}\left(\rho_{\psi, c} ; \rho_{\psi^{\prime}, c}\right)=\tau_{\mathrm{A}} \frac{1}{n A(\Omega)},
$$

where $\tau_{\mathrm{A}}$ denotes the probability of proposing move type A. The probability $q_{\mathrm{A}^{\prime}}$ for the reverse move $\mathrm{A}^{\prime}$ to be generated is equal to the probability of choosing to delete an interior 
vertex in $\psi$, times the probability of picking that vertex from the $n+1$ vertices in $\psi^{\prime}$ i.e.,

$$
q_{\mathrm{A}^{\prime}}\left(\rho_{\psi^{\prime}, c} ; \rho_{\psi, c}\right)=\tau_{\mathrm{A}^{\prime}} \frac{1}{n+1}
$$

The Jacobian term $\left|\partial f\left(\rho_{\psi, c}, u\right) / \partial\left(\rho_{\psi, c}, u\right)\right|$ needed for computing the acceptance probability is simply one, since $f\left(\rho_{\psi, c}, u\right)=\left(\rho_{\psi, c}, u\right)$. Thus, the posterior ratio in (9) becomes

$$
\frac{p_{\Omega, C}\left(\rho_{\psi^{\prime}, c}, \nu, L, \sigma \mid y\right)}{p_{\Omega, C}\left(\rho_{\psi, c}, \nu, L, \sigma \mid y\right)}=\frac{|C(\psi)| \lambda_{\Omega}^{n+1} \lambda_{\partial \Omega}^{m} \exp \left(-\theta_{\zeta} \zeta\left(\rho_{\psi^{\prime}, c}\right)-G\left(\rho_{\psi^{\prime}, c} ; \Sigma\right)\right)}{\left|C\left(\psi^{\prime}\right)\right| \lambda_{\Omega}^{n} \lambda_{\partial \Omega}^{m} \exp \left(-\theta_{\zeta} \zeta\left(\rho_{\psi, c}\right)-G\left(\rho_{\psi, c} ; \Sigma\right)\right)} .
$$

The underlying polygonal model is changed by this update so the overall length of the discontinuity set is most likely changed. However, the colouring is not changed, so $\left|C\left(\psi^{\prime}\right)\right|=$ $|C(\psi)|$ and densities cancel between prior measures up to factor of $\lambda_{\Omega}$ for the extra interior vertex in $\psi^{\prime}$. Hence, by (9), the corresponding acceptance probability becomes

$$
\alpha_{\mathrm{A}}\left(\rho_{\psi, c} ; \rho_{\psi^{\prime}, c}\right)=\min \left\{1, \mathbf{1}\left(\psi^{\prime} \in \mathbf{\Psi}_{\Omega}\right) \lambda_{\Omega} A(\Omega) \frac{\tau_{\mathrm{A}^{\prime}}}{\tau_{\mathrm{A}}} \frac{n}{n+1} \frac{\exp \left[-\theta_{\zeta} \zeta\left(\rho_{\psi^{\prime}, c}\right)-G\left(\rho_{\psi^{\prime}, c} ; \Sigma\right)\right]}{\exp \left[-\theta_{\zeta} \zeta\left(\rho_{\psi, c}\right)-G\left(\rho_{\psi, c} ; \Sigma\right)\right]}\right\} .
$$

The death of a vertex is performed similarly, with one of the $n$ interior vertices being proposed for deletion, a move which is subsequently accepted with probability

$$
\alpha_{\mathrm{A}^{\prime}}\left(\rho_{\psi, c} ; \rho_{\psi^{\prime}, c}\right)=\min \left\{1, \mathbf{1}\left(\psi^{\prime} \in \Psi_{\Omega}\right) \frac{1}{\lambda_{\Omega} A(\Omega)} \frac{\tau_{\mathrm{A}}}{\tau_{\mathrm{A}^{\prime}}} \frac{n}{n-1} \frac{\exp \left[-\theta_{\zeta} \zeta\left(\rho_{\psi^{\prime}, c}\right)-G\left(\rho_{\psi^{\prime}, c} ; \Sigma\right)\right]}{\exp \left[-\theta_{\zeta} \zeta\left(\rho_{\psi, c}\right)-G\left(\rho_{\psi, c} ; \Sigma\right)\right]}\right\} .
$$

\subsection{Metropolis-coupled Markov chain Monte Carlo}

The Metropolis-Hastings and reversible jump updates will produce a Markov chain with the required stationary distribution. However, the resulting chain may be slow to move around the state space so that large run lengths are required in order to obtain reliable inference. To improve the speed with which the state space is traversed (often termed the mixing rate), many simulation techniques have been suggested. For example, MCMCMC (Geyer, 1991), simulated tempering (Marini and Parisi, 1992; Geyer and Thompson, 1995), multigrid Monte Carlo (MGMC) methods (Goodman and Sokal, 1989) and simulated sintering (Liu and Sabatti, 1998) have all been proposed as mechanisms for improving Markov chain mixing. In this paper, we combine the MCMCMC and MGMC simulation algorithms to obtain the Metropolis-coupled multigrid Markov chain Monte Carlo simulation algorithm described below. Actually, Higdon et al. (2001) consider a similar approach using low-level multivariate Gaussian priors on the different grids. This contrasts our high-level approach where the prior distribution is placed on the space of polygons.

Let $\Pi=\left\{\pi_{1}, \pi_{2}, \ldots, \pi_{d}\right\}$ denote a family of interrelated stationary distributions and suppose $d$ Markov chains are run in parallel, each with a different stationary distribution from $\Pi$. The method works by running all chains simultaneously, but occasionally proposing to swap the states of two randomly chosen chains. Suppose $\pi_{1}$ is the (posterior) distribution of interest and that states from chain $i$ that can be swapped between chains are spaced $N_{i}$ iterations apart. This allows for the fact that the different chains may mix at different rates so that autocorrelations between states within some chains will die out more slowly than in others.

Let $\rho_{t}^{(i)}$ denote the state of the $i$ th chain (with stationary distribution $\pi_{i}$ ) at iteration $t$ and suppose that we propose to swap states between chains $i$ and $i+1$. Then the probability 
of accepting the proposed swap is simply

$$
\min \left\{1, \frac{\pi_{i}\left(\rho_{N_{i+1} t_{i}}^{(i+1)}\right) \pi_{i+1}\left(\rho_{N_{i} t_{i}}^{(i)}\right)}{\pi_{i}\left(\rho_{N_{i} t_{i}}^{(i)}\right) \pi_{i+1}\left(\rho_{N_{i+1} t_{i}}^{(i+1)}\right)}\right\} .
$$

We apply the Metropolis-coupled MCMC algorithm described above by taking $d=4$. When evaluating the likelihood function there are two issues to consider: (1) the number of sampling locations on the surface and (2) the coarseness of the grid used to approximate the profile in consideration. We use different numbers of sampling locations and grid coarseness to create our four target densities. We let $\left\{\rho_{t}^{(1)}\right\}$ denote the chain of the full data evaluated by using a grid with cell size 1-by-1 metres i.e., the approximating matrix is 45 -by-450. This is the density of interest. The underlying process is also sampled in all possible locations on the profile. For the second chain, we take a slightly coarser grid with cell size 5-by-3 metres i.e., a 15-by-90 matrix is used to approximate the profile. The third process is based upon the same grid, but we sample observations only every 30 metres. The final process uses the same sample locations and uses an even coarser grid with cell sizes of 9-by-15 metres.

\subsection{Convergence properties}

Let $\rho_{t}=\left\{\rho_{(t-1) N_{1}+1}^{(1)}, \ldots, \rho_{t N_{1}}^{(1)}, \ldots, \rho_{(t-1) N_{d}+1}^{(d)}, \ldots, \rho_{t N_{d}}^{(d)}\right\}$ denote the overall process with

stationary distribution $\bigotimes_{i=1}^{d} \pi_{i}^{N_{i}}$ (Geyer, 1991). Obviously, for any two polygonal models of fixed vertex sets, there is a finite sequence of operations linking the two models. This, in combination with the likelihood being bounded below from 0 means that the Markov chain is irreducible and Harris recurrent (Robert and Casella, 1999, Proposition 4.4.9). Consequently the chain will converge (in total variation norm) to a unique stationary distribution. The Markov chain described in this section was implemented and tested by setting $G$, defined in (5), equal to zero and thereby performing several simulations from the prior varying only the branching probabilities. Since estimates from these different runs are similar, we assume that the chain therefore samples from the correct distribution.

\section{Analysis of field data from the Ølst formation}

In this section, we consider the performance of our approach on the 150 metre long data segment shown in Figure 3(a). To avoid boundary effects we propose augmenting the profile of interest into a 450 metre long and 45 metre deep profile $\rho$. The reconstruction for the segment of interest is then based upon apparent resistivities predicted for the middle segment by the forward operator $K$ derived in Section 2. A large number of colours in $\mathcal{J}$ is expected to lead to slow convergence and it is therefore important to recover good estimates of the colours to be used in the MCMC simulation. A natural approach would be to consider a mixture distribution over possible colour values. However, we have briefly experimented with this technique, and found that the additional degrees of freedom lead to very slow convergence properties. In the reconstruction we present here we instead condition upon the number of colours and use a fixed set of colour values based upon prior knowledge and pilot tuning of the MCMC algorithm. A colour set $\mathcal{J}$ based upon 18 colours ranging from $1 / 2$ to 2000 is used for the reconstruction, see Table 1 for details. The colours are chosen in order to cover the resistivities of the earth materials believed to be present in the Ølst 
Table 1. Initial values used in the MCMC reconstruction algorithm.

\begin{tabular}{|cccccccccc|}
\hline \multicolumn{10}{|c|}{ Colour values } \\
\hline $1 / 2$ & 1 & 3 & 5 & 10 & 20 & 30 & 40 & 60 \\
80 & 100 & 300 & 500 & 750 & 1000 & 1200 & 1500 & 2000 \\
\hline \multicolumn{10}{|c}{ Prior model/initial parameters } \\
\cline { 2 - 8 } & $\lambda$ & $\theta_{\zeta}$ & $\sigma$ & $L$ & $\nu$ & $b_{\sigma}$ & $b_{L}$ & $b_{\nu}$ \\
& 5 & $1 / 10$ & 1 & 100 & 1 & 1 & 4 & 10 & \\
\hline
\end{tabular}

Table 2. The resulting posterior means, standard deviances and $95 \%$ credible intervals for the parameters in the MCMCMC simulation.

\begin{tabular}{|crrrr|}
\hline & & & \multicolumn{2}{c|}{$95 \%$ C.I. } \\
\cline { 4 - 5 } Parameter & Post. mean & St.d. & Lower & Upper \\
\hline$\sigma$ & 0.4903 & 0.0104 & 0.4705 & 0.5104 \\
$L$ & 23.9793 & 1.4882 & 21.6018 & 26.3866 \\
$\nu$ & 0.1621 & 0.0241 & 0.1202 & 0.2070 \\
\hline
\end{tabular}

Formation. The overall mean of the data segment is about $100 \Omega \cdot \mathrm{m}$ which we therefore take as the reference resistivity distribution i.e., $\rho_{\text {ref }}=100$.

For the simulation we choose $\tau_{\mathrm{A}}=\tau_{\mathrm{A}^{\prime}}=1 / 16, \tau_{\mathrm{B}}=\tau_{\mathrm{B}^{\prime}}=1 / 20, \tau_{\mathrm{C}}=\tau_{\mathrm{C}^{\prime}}=1 / 80$, $\tau_{\mathrm{D}}=3 / 8, \tau_{\mathrm{E}}=3 / 10$ and $\tau_{\mathrm{F}}=3 / 40$, so that the chain spends more time translating and recolouring the polygons than inserting and deleting them. These settings are likely to be suitable in most contexts. For the prior on the number of boundary vertices and the number of interior vertices in $\psi$ we choose intensities $\lambda_{\Omega}=\lambda^{2}$ and $\lambda_{\partial \Omega}=\lambda$ with $\lambda=8$. The tuning parameter $\theta_{\zeta}$ was chosen to be large i.e., $\theta_{\zeta}=1 / 10$, so that the colour of neighbouring polygonal faces is highly correlated in the prior. For the updating of the variance parameters we choose centered proposal kernels i.e., $a_{\sigma}=b_{\sigma} \tau$ with $b_{\sigma}=1 ; a_{L}=b_{L} L$ with $b_{L}=4$; and $a_{\nu}=b_{\nu} \nu$ with $b_{\nu}=10$.

For the four different MCMC simulations we choose homogeneous resistivity distributions of $10,50,100$ and $500 \Omega \cdot \mathrm{m}$ as initial distributions each started with $\sigma=1, L=100$ and $\nu=1$ as initial parameters. Swaps in $\pi_{1}$ were attempted at every 20 iterations i.e., $N_{1}=20$. The remaining chains had $N_{2}=50, N_{3}=200$ and $N_{4}=500$. The master process was run for 150000 iterations, while the three remaining processes were kept running while the master process was active i.e., for 375000,1500000 and 3750000 iterations, respectively. Swaps between chains $i$ and $i+1, i=1,2,3$, were attempted at random. Figure 10(a) displays the posterior potential for an ordinary run of the MCMC algorithm without coupling and a run of the coupled MCMC algorithm. It is evident that the coupled simulation algorithm improves dramatically upon the sampling performance of $\rho^{(1)}$ based only upon MCMC and RJMCMC moves.

In the absence of a suitable test for Markov chain convergence, we look for stationarity in selected output statistics. Figure 10(a) displays trace plots for the posterior potential $G(\rho ; \sigma, \nu, L)$. Trace plots for other statistics exhibit similar behaviour and we therefore assume that the chain achieves stationarity within the first 75000 iterations. The remaining 75000 iterations are used for posterior inference and a summary of the results is presented in Table 2. The autocorrelation function (ACF) for the coupled MCMC algorithm, see Figure 10(b), was estimated for the posterior potential. The ACF provides a useful tool for investigating serial dependence in stationary time series data, as the presence of serial correlation is revealed by a slowly decaying ACF. The autocorrelations for a stationary time 

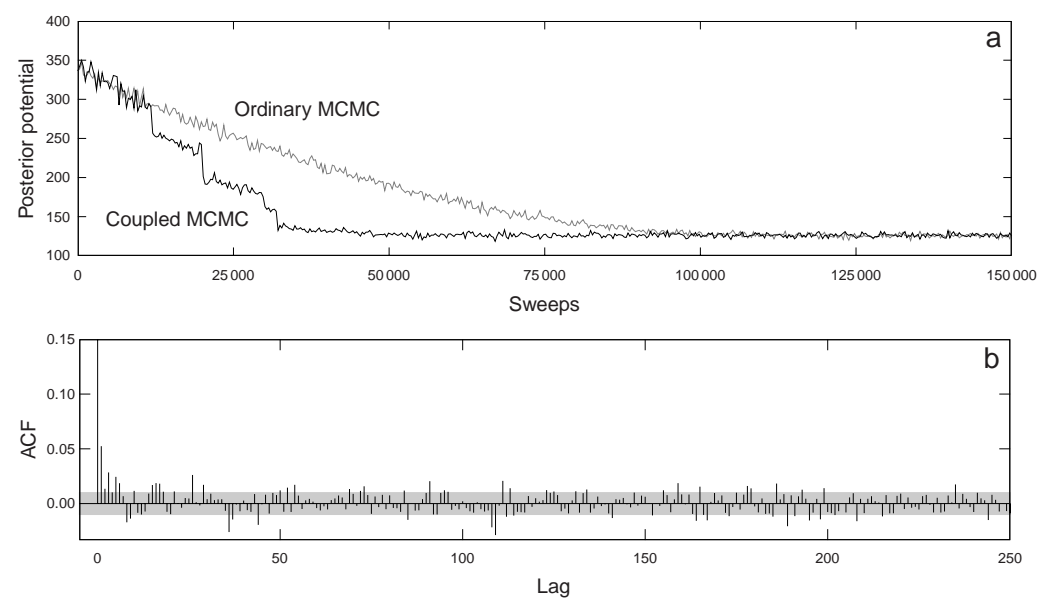

Fig. 10. Sequence of statistics observed in the polygonal model during the MCMC reconstruction procedure. (a) The posterior potential and (b) the estimated ACF for the coupled algorithm measured in MCMC simulations. Superimposed in gray is the $95 \%$ confidence interval from the estimated asymptotic variance.

series with no or only little serial dependence, damp down to zero very quickly for increasing lag. The ACF in Figure 10(b) exhibits similar behavior and we therefore conclude that only little serial dependence is present in the chain. However, one must note that the $G$ statistic need not necessarily be the worst mixing statistic. Nevertheless, ACFs were computed for several other statistics and they exhibited similar behavior.

Some sort of summary is needed for the interpretation of the reconstruction. Figure 11(a) provides the maximum a posteriori estimate in which the profile is clearly segmented into five well-defined sedimentary areas. It is not possible to construct an average of the graphs encountered during the MCMC simulation. However, as suggested by Nicholls (1998), we may sample points uniformly on the discontinuity set of colourings sampled from the posterior in order to assess the variability of the transitions between distinct earth materials, see Figure 11(b). As suggested by Møller et al. (2001), the vertical shear between clay and sand is even sharper than predicted by the MCD inversion estimate. Moreover, the samples from the discontinuity set may indicate that the transitions are rather well-determined. This is also supported by the posterior mean resistivity distribution within each cell and its corresponding coefficient of variation given by the standard deviation in each pixel divided by its corresponding mean, see Figure 11(c) and (d) for details. The reconstruction obtained by the MCD technique clearly indicated three sedimentary areas and a rather diffuse isolated area, see Figure 3(b). Our approach revealed, besides a fourth well-defined sedimentary area, a smaller and better identified isolated area. However, this area is not as well determined as the remaining areas. This may be due to its small size, however, the near-surface low resistivity area may provide low-quality measurements and thereby distort the reconstruction.

\section{Discussion}

In this paper, we develop and discuss a practical Bayesian method for the inversion of an illposed inverse problem. A coloured polygonal model serves as a flexible and comprehensive 

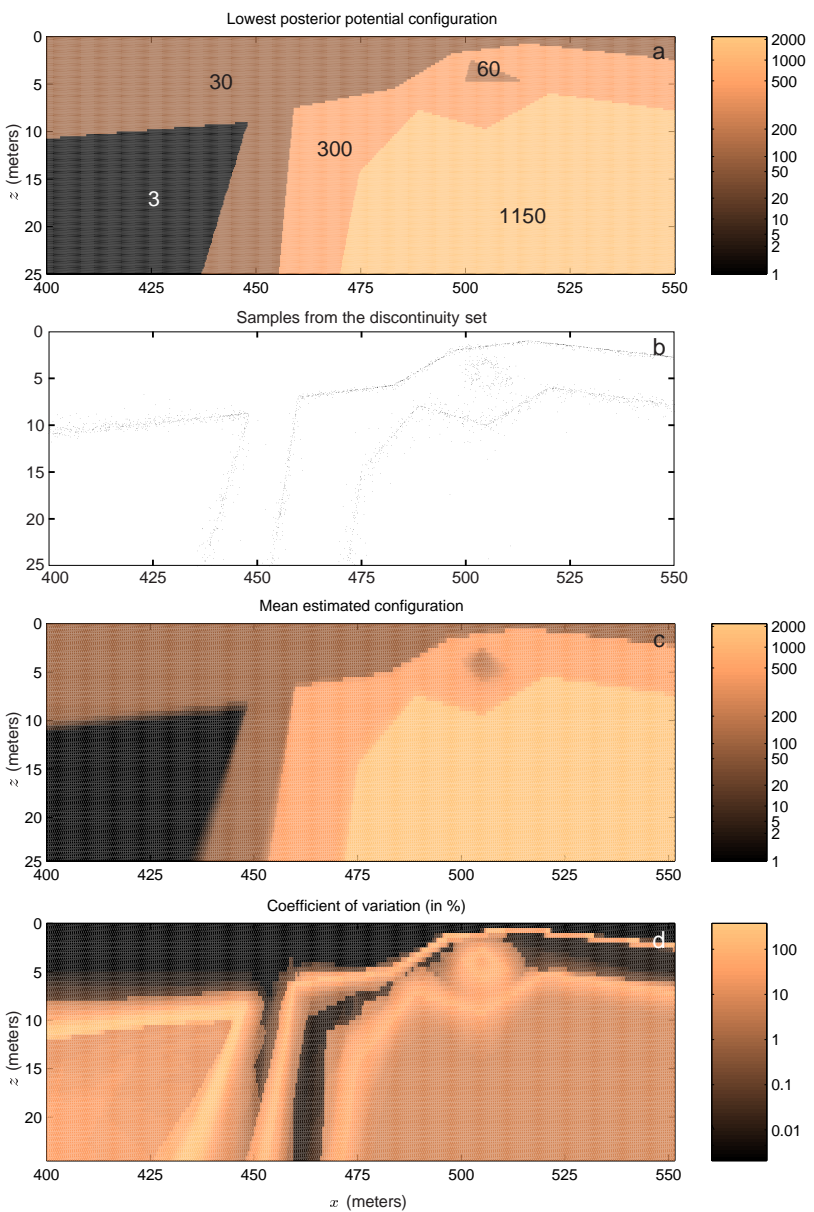

Fig. 11. (a) The state with the lowest posterior potential sampled during the MCMC simulation; (b) 2500 points sampled uniformly from the discontinuity set; (c) the estimated posterior mean profile; and $(d)$ the estimated coefficient of variation of the profile distribution.

model for large-scale mapping of the resistivity distribution within a 2-dimensional profile. The reversible jump MCMC algorithm is used to obtain the reconstruction. This simulation algorithm permits a state space of varying dimensionality and therefore enables us to draw inference on the number of polygonal faces or the number of edges, for example.

However, the simulation algorithm proposed here can be computer-intensive due to the time-consuming updating process. The Metropolis-coupled MCMC technique was exploited to induce better mixing Markov chains which reduces the necessary run length. Usually the chains are defined on the same state space. However, we adopt the multigrid Markov chain technique and propose swapping the states of Markov chains defined on dissimilar state spaces. The efficiency of this geophysical reconstruction simulation algorithm was compared to the ordinary reversible jump MCMC algorithm and faster convergence was obviously achieved.

One drawback associated with the proposed method is the use of an approximate forward solver. If an exact solver were used, we would probably obtain even more reliable 
reconstructions. However, exact forward solvers are even more computer-intensive than the approximations, and the simulation algorithm could become very slow. However, a combined Metropolis algorithm may be constructed to provide good candidate models for the exact forward solver i.e., models that are most likely to be accepted.

The analysis could be extended in a number of ways. For example, we could remove the condition that the values of $\lambda_{\partial \Omega}, \lambda_{\Omega}$ and $\theta_{\zeta}$ be fixed. In which case, we would place hyper-priors on these parameters though this may slow convergence. In addition, we could generalise the error process in order to better model small scale variation and this is the focus of current research. Current interest also focusses on the modelling of the topography of the Earth, as hilly topography will act to disperse current flow on hills and concentrate current flow in valleys, as well as the incorporation of borehole information. Moreover, a $3 \mathrm{D}$ reconstruction algorithm is under consideration.

\section{Acknowledgments}

We would like to thank Ingelise Møller, Geological Survey of Denmark and Greenland, Denmark, and Bo Holm Jacobsen, Department of Geophysics, Aarhus University, Denmark, for providing the Ølst data and the forward solver. The research has been supported by the European Union's research network "Statistical and Computational Methods for the Analysis of Spatial Data. ERB-FMRX-CT96-0095". SPB was supported by EPSRC, The Engineering and Physical Sciences Research Council, UK, and MBH was supported by MaPhySto, Centre for Mathematical Physics and Stochastics, funded by a grant from The Danish National Research Foundation.

\section{A. The Fréchet derivative for 2-dimensional resistivity data}

Let $I$ denote the current induced into the Earth at $(0,0,0)$ and let $\left(x_{s}, 0,0\right)$ denote the lateral focus for the electrical potential $V_{p p}$ in any pole-pole electrode configuration $s$. The 3-dimensional form of the Fréchet derivative for the homogeneous half-space is then given by (cf. Boerner and West, 1989)

$$
\frac{\partial V_{p p}\left(x_{s}\right)}{\partial \rho(x, y, z)}=\frac{I}{4 \pi^{2}} \frac{x\left(x-x_{s}\right)+y^{2}+z^{2}}{\left(x^{2}+y^{2}+z^{2}\right)^{3 / 2}\left(\left(x-x_{s}\right)^{2}+y^{2}+z^{2}\right)^{3 / 2}} .
$$

The 2-dimensional form of the Fréchet derivative is obtained by analytical integration of (10) along strike i.e., in the $y$-direction. Let $\mathrm{K}$ and $\mathrm{E}$ denote the complete elliptical integrals of the first and second kind, respectively. Hence

$$
\frac{\partial V_{p p}\left(x_{s}\right)}{\partial \rho(x, z)}= \begin{cases}\frac{I}{2 \pi^{2} \beta}\left[\frac{\mathrm{K}\left(q_{1}\right)-\mathrm{E}\left(q_{1}\right)}{\beta^{2}-\alpha^{2}}-x_{s} x \frac{\left(\beta^{2}+\alpha^{2}\right) \mathrm{E}\left(q_{1}\right)-2 \alpha^{2} \mathrm{~K}\left(q_{1}\right)}{\alpha^{2}\left(\beta^{2}-\alpha^{2}\right)}\right] & \text { if } x<a / 2 \\ \frac{I}{16 \pi} \frac{2 z^{2}-x^{2}}{\left(x^{2}+z^{2}\right)^{5 / 2}} & \text { if } x=a / 2 \\ \frac{I}{2 \pi^{2} \alpha \beta^{2}}\left[\frac{\alpha^{2} \mathrm{E}\left(q_{2}\right)-\beta^{2} \mathrm{~K}\left(q_{2}\right)}{\alpha^{2}-\beta^{2}}-x_{s} x \frac{\left(\beta^{2}+\alpha^{2}\right) \mathrm{E}\left(q_{2}\right)-2 \beta^{2} \mathrm{~K}\left(q_{2}\right)}{\left(\alpha^{2}-\beta^{2}\right)^{2}}\right] & \text { else, }\end{cases}
$$

where $\alpha^{2}=x^{2}+z^{2}, \beta^{2}=\left(x-x_{s}\right)^{2}+z^{2}, q_{1}=\sqrt{\left(\beta^{2}-\alpha^{2}\right)} / \beta$ and $q_{2}=\sqrt{\left(\alpha^{2}-\beta^{2}\right)} / \alpha$. Finally, the electrical potential for a four-electrode configuration is given by a sum of four pole-pole potentials, that is, the total 2-dimensional form of the Fréchet derivative becomes

$$
\frac{\partial V_{s}\left(x_{s}\right)}{\partial \rho(x, z)}=\frac{\partial V_{p p}\left(x_{M}-x_{A}\right)}{\partial \rho\left(x-x_{A}, z\right)}-\frac{\partial V_{p p}\left(x_{N}-x_{A}\right)}{\partial \rho\left(x-x_{A}, z\right)}-\frac{\partial V_{p p}\left(x_{M}-x_{B}\right)}{\partial \rho\left(x-x_{B}, z\right)}+\frac{\partial V_{p p}\left(x_{N}-x_{B}\right)}{\partial \rho\left(x-x_{B}, z\right)} .
$$




\section{B. Detailed move descriptions}

In this Appendix we provide detailed descriptions of the move-types used in the reconstruction algorithm.

$A / A^{\prime}:$ Vertex birth and death

The birth and death moves are described in detail in Section 3.2.

\section{$B / B^{\prime}$ : boundary polygonal birth and death}

Suppose that the current configuration $\rho_{\psi, c}$ consists of $n$ interior vertices and $m$ boundary vertices. Then, a new boundary polygon is introduced to $\psi$ by joining a straight line between two uniformly and independently distributed vertices $u_{1}$ and $u_{2}$ on the boundary $\partial \Omega$ i.e. $\left(u_{1}, u_{2}\right)$ has density $1 / L(\partial \Omega)^{2}$. If the edge $e=u_{1} u_{2}$ crosses any other in $\psi$, then the move is automatically rejected. Otherwise, the inserted edge splits a polygonal face into two, each of which is assigned a new colour uniformly from $\mathcal{J}$. The edge $e$ can be generated in two different ways, so the generation probability becomes

$$
q_{\mathrm{B}}\left(\rho_{\psi, c} ; \rho_{\psi^{\prime}, c^{\prime}}\right)=\tau_{\mathrm{B}} \frac{2}{|\mathcal{J}|^{2} L(\partial \Omega)^{2}} .
$$

Boundary polygonal death is chosen with probability $\tau_{\mathrm{B}^{\prime}}$. A boundary vertex $v$ is chosen uniformly among the $m+2$ existing boundary vertices in $\psi^{\prime}$. If $v=u_{1}$ or $v=u_{2}$, then we consider the state $\rho_{\psi, c}$ formed from $\rho_{\psi, c}^{\prime}$ by deleting the edge $e$ and assigning the faces incident to $e$ a new common colour. The generation probability for the reverse move $\mathrm{B}^{\prime}$ is then

$$
q_{\mathrm{B}^{\prime}}\left(\rho_{\psi^{\prime}, c^{\prime}} ; \rho_{\psi, c}\right)=\tau_{\mathrm{B}^{\prime}} \frac{2}{(m+2)|\mathcal{J}|} .
$$

The Jacobian equals one, so combining the generation probabilities the acceptance probability for boundary polygonal birth becomes

$$
\alpha_{\mathrm{B}}\left(\rho_{\psi, c} ; \rho_{\psi^{\prime}, c^{\prime}}\right)=\min \left\{1, \mathbf{1}\left(\psi^{\prime} \in \mathbf{\Psi}_{\Omega}\right) \frac{\tau_{\mathrm{B}^{\prime}}}{\tau_{\mathrm{B}}} \frac{|\mathcal{J}| L(\partial \Omega)^{2} \lambda_{\partial \Omega}^{2}}{m+2} \frac{\exp \left[-\theta_{\zeta} \zeta\left(\rho_{\psi^{\prime}, c^{\prime}}\right)-G\left(\rho_{\psi^{\prime}, c^{\prime}} ; \Sigma\right)\right]}{\exp \left[-\theta_{\zeta}\left(\zeta\left(\rho_{\psi, c}\right)-G\left(\rho_{\psi, c} ; \Sigma\right)\right]\right.}\right\} .
$$

The acceptance probability for the reverse move to be performed is easily found to be

$$
\alpha_{\mathrm{B}^{\prime}}\left(\rho_{\psi, c} ; \rho_{\psi^{\prime}, c^{\prime}}\right)=\min \left\{1, \mathbb{1}\left(\psi^{\prime} \in \Psi_{\Omega}\right) \frac{\tau_{\mathrm{B}}}{\tau_{\mathrm{B}^{\prime}}} \frac{m}{|\mathcal{J}| L(\partial \Omega)^{2} \lambda_{\partial \Omega}^{2}} \frac{\exp \left[-\theta_{\zeta} \zeta\left(\rho_{\psi^{\prime}, c^{\prime}}\right)-G\left(\rho_{\psi^{\prime}, c^{\prime}} ; \Sigma\right)\right]}{\exp \left[-\theta_{\zeta} \zeta\left(\rho_{\psi, c}\right)-G\left(\rho_{\psi, c} ; \Sigma\right)\right]}\right\} .
$$

\section{$C / C^{\prime}$ : interior polygonal birth and death}

The births and deaths of interior polygons are performed along similar lines as the birth and death updates for boundary polygons. If we assume $\psi$ consists of $n$ interior vertices and an interior polygonal birth is attempted, then three vertices $\left(u_{1}, u_{2}, u_{3}\right)$ are sampled uniformly and independently in $\Omega$. Note, the three vertices may be re-labeled in six different ways. If the edges of the polygonal face $\left(u_{1}, u_{2}, u_{3}\right)$ cross any others in $\psi$, then it is rejected, otherwise it is assigned a colour uniformly from the colours in $\mathcal{J}$. The generation probability for this move is then

$$
q_{\mathrm{C}}\left(\rho_{\psi, c} ; \rho_{\psi^{\prime}, c^{\prime}}\right)=\tau_{\mathrm{C}} \frac{6}{|\mathcal{J}| A(\Omega)^{3}} .
$$

The generation probability for the reverse move $\mathrm{C}^{\prime}$ is equal to the probability of choosing to delete an interior polygonal face, times the probability of picking any of the three vertices of all the $n+3$ vertices in $\psi^{\prime}$ i.e.,

$$
q_{\mathrm{C}^{\prime}}\left(\rho_{\psi^{\prime}, c^{\prime}} ; \rho_{\psi, c}\right)=\tau_{\mathrm{C}^{\prime}} \frac{3}{n+3}
$$


Note that the death of an interior polygonal face does not require a new colour to be sampled in contrast to the boundary polygonal death, since the embedding polygonal face maintains its colouring. The corresponding acceptance probability then becomes

$$
\alpha_{\mathrm{C}}\left(\rho_{\psi, c} ; \rho_{\psi^{\prime}, c^{\prime}}\right)=\min \left\{1, \mathbf{1}\left(\psi^{\prime} \in \mathbf{\Psi}_{\Omega}\right) \frac{\tau_{\mathrm{C}^{\prime}}}{\tau_{\mathrm{C}}} \frac{A(\Omega)^{3} \lambda_{\Omega}^{3}|\mathcal{J}|}{2(n+3)} \frac{\exp \left[-\theta_{\zeta} \zeta\left(\rho_{\psi^{\prime}, c^{\prime}}\right)-G\left(\rho_{\psi^{\prime}, c^{\prime}} ; \Sigma\right)\right]}{\exp \left[-\theta_{\zeta}\left(\zeta\left(\rho_{\psi, c}\right)-G\left(\rho_{\psi, c} ; \Sigma\right)\right]\right.}\right\} .
$$

Similarly, the acceptance probability for the reverse move is easily found to be

$$
\alpha_{\mathrm{C}^{\prime}}\left(\rho_{\psi, c} ; \rho_{\psi^{\prime}, c^{\prime}}\right)=\min \left\{1, \mathbf{1}\left(\psi^{\prime} \in \mathbf{\Psi}_{\Omega}\right) \frac{\tau_{\mathrm{C}}}{\tau_{\mathrm{C}^{\prime}}} \frac{2 n}{A(\Omega)^{3} \lambda_{\Omega}^{3}|\mathcal{J}|} \frac{\exp \left[-\theta_{\zeta} \zeta\left(\rho_{\psi^{\prime}, c^{\prime}}\right)-G\left(\rho_{\psi^{\prime}, c^{\prime}} ; \Sigma\right)\right]}{\exp \left[-\theta_{\zeta}\left(\zeta\left(\rho_{\psi, c}\right)-G\left(\rho_{\psi, c} ; \Sigma\right)\right]\right.}\right\} .
$$

\section{D: Recolouring polygonal faces}

This move type is chosen with probability $\tau_{\mathrm{D}}$. We choose a polygonal face $f_{i}$ uniformly from the $f_{\psi}$ polygons faces in $\psi$ and let $\mathcal{J}_{i}$ denote the colour of the picked polygon. A new colour, $\mathcal{J}_{j}$ say, is chosen uniformly from $\mathcal{J} \backslash \mathcal{J}_{i}$ and we consider the candidate state $\rho_{\psi, c^{\prime}}$ obtained from $\rho_{\psi, c}$ by setting $c_{f}=\mathcal{J}_{j}$. The generation probability for this move is simply

$$
q_{\mathrm{D}}\left(\rho_{\psi, c} ; \rho_{\psi, c^{\prime}}\right)=\tau_{\mathrm{D}} \frac{1}{(|\mathcal{J}|-1)|\mathcal{J}|}
$$

Since this proposal is symmetric, the acceptance probability simplifies to

$$
\alpha_{\mathrm{D}}\left(\rho_{\psi, c} ; \rho_{\psi, c^{\prime}}\right)=\min \left\{1, \exp \left[\theta_{\zeta}\left(\zeta\left(\rho_{\psi, c}\right)-\zeta\left(\rho_{\psi, c^{\prime}}\right)\right)+G\left(\rho_{\psi, c} ; \Sigma\right)-G\left(\rho_{\psi, c^{\prime}} ; \Sigma\right)\right]\right\} .
$$

In practice, we consider each face in turn, so that each may be updated within a single iteration.

\section{E: Translating polygons}

The translation of polygons is described in detail in Section 3.1.

\section{F: Shifting edges in polygons}

With probability $\tau_{\mathrm{F}}$ we shift two of the edges in $\psi$. Two vertices $\left(u_{1}, v_{1}\right)$ are picked uniformly from the $n$ vertices in $\psi$. Let $u_{2}$ denote a neighbouring vertex of $u_{1}$, picked at random. Likewise, let $v_{2}$ denote a neighbouring vertex of $v_{1}$, picked at random. Finally, let $\psi^{\prime}$ denote the polygonal model in which edges $\left(u_{1}, u_{2}\right)$ and $\left(v_{1}, v_{2}\right)$ in $\psi$ are replaced by edges $\left(u_{1}, v_{1}\right)$ and $\left(u_{2}, v_{2}\right)$. Then the faces in $\psi^{\prime}$ are all assigned new colours from $\mathcal{J}$. If we let $f_{\psi}$ denote the number of polygonal faces in $\psi$, then the generation probability is

$$
q_{\mathrm{F}}\left(\rho_{\psi^{\prime}, c^{\prime}} ; \rho_{\psi, c}\right)=\tau_{\mathrm{F}} \frac{1}{2(n+m)|\mathcal{J}|^{f_{\psi}}},
$$

since $f_{\psi}=f_{\psi^{\prime}}$. The acceptance probability $\alpha_{\mathrm{F}}$ then becomes identical to $\alpha_{\mathrm{D}}$ and $\alpha_{\mathrm{E}}$.

\section{References}

Andersen, K. E., Brooks, S. P. and Hansen, M. B. (2001). A Bayesian approach to crack detection in electrically conducting media. Inverse Problems 17, 121 - 136.

Arak, T. and Surgailis, D. (1989). Markov fields with polygonal realisations. Probability Theory Related Fields 80, 543 - 579.

Arak, T., Clifford, P. and Surgailis, D. (1993). Point-based polygonal models for random graphs. Advances of Applied Probability 25, 348 - 372. 
Boerner, D. E. and West, G. F. (1989). Fréchet derivatives and single scattering theory. Geophysical Journal International 98, 385 - 390 .

Brooks, S.P. (1998). Markov chain Monte Carlo method and its application. The Statistician 47, $69-100$.

Brown, W. G. (1964). Enumeration of triangulations of the disk. Proceedings of the London Mathematical Society 14, $746-768$.

Christensen, N.B. and Sørensen, K.I. (1998). Surface and borehole electric and electromagnetic methods for hydrogeological investigations. European Journal of Environmental and Engineering Geophysics 3, $75-90$.

Daily, W., Ramirez, A., LaBrecque, D. and Barber, W. (1995). Electrical resistance tomography at the Oregon Graduate Institute. Journal of Geophysics 33, 227 - 237.

Edwards, L. S. (1977). A modified pseudosection for resistivity and IP. Geophysics 42, 275 - 283.

Fox, C. and Nicholls, G. K. (1997). Sampling conductivity images via MCMC. In: The art and science of Bayesian image analysis, Proceedings of the Leeds Annual Statistics Research Workshop (eds. K. V. Mardia, C. A. Gill and R. Aykroyd), Leeds University Press, $91-100$.

Geyer, C. J. (1991). Markov chain Monte Carlo maximum likelihood. In: Computing Science and Statistics: Proceedings of the 23rd Symposium on the Interface, 156-163.

Geyer, C.J. and Thompson, E.A. (1995). Annealing Markov chain Monte Carlo with applications to ancestral inference. J. Am. Statist. Ass. 90, $909-920$.

Goodman, J. and Sokal, A. D. (1989). Multigrid Monte Carlo method. Conceptual foundations. Physical Review D 40, 2035 - 2072.

Green, P. (1995). Reversible jump Markov chain Monte Carlo computation and Bayesian model determination. Biometrika 82, 711 - 32 .

Hastings, W.K. (1970). Monte Carlo sampling methods using Markov chains and their applications. Biometrika 57, 97 - 109.

Heilmann-Clausen, C., Nielsen, O.B. and Gersner, F. (1985). Lithostatigraphy and depositional environments in the Upper Paleocene and Eocene of Denmark. Bulletin of the Geological Society of Denmark 33, $287-323$.

Higdon, D., Lee, H., and Bi, Z. (2001). A Bayesian approach to characterizing uncertainty in inverse problems using coarse and fine scale information. Technical report, Duke University, Institute of Statistics and Decision Sciences.

Jacobsen, B. H. (1993). Practical methods of a priori covariance specification for geophysical inversion. In: Proceedings of the Interdisciplinary Inversion Workshop 2 (ed. K. Mosegaard), University of Copenhagen, 1 - 10 .

Kaipio, J. P., Kolehmainen, V., Somersalo, E. and Vauhkonen, M. (2000). Statistical inversion and Monte Carlo sampling methods in electrical impedance tomography. Inverse Problems 16, 1487 -1522 .

Li, Y. and Oldenburg, D. W. (1992). Approximate inverse mappings in DC resistivity problems. Geophysical Journal International 109, 343 - 362. 
Liu, J. S. and Sabatti, C. (1998). Simulated sintering: Markov chain Monte Carlo with spaces of varying dimensions (with discussions). In: Bayesian Statistics 6 (eds. J. M. Bernado, J. O. Berger, A. P. Dawid and A. F. M. Smith), Oxford University Press, $389-413$.

Loke, M. H. and Barker, R. D. (1995). Rapid least-squares inversion of apparent resistivity pseudosections by a quasi-Newton method. Geophysics 60, 1682 - 1690.

Marini, E. and Parisi, G. (1992). Simulated tempering: a new Monte Carlo scheme. Europhysics Letters 19, 451 - 458.

Matérn, B. (1986). Spatial Variation. Springer-Verlag, Berlin, 2nd edition.

McGillivray, P. R. (1992). Forward modeling and inversion of DC resistivity and MMR data. Ph.D. thesis, The University of British Columbia, Vancouver, Canada.

Metropolis, N., Rosenbluth, A. W., Rosenbluth, M. N., Teller, A. H. and Teller, E. (1953). Equations of state calculations by fast computing machines. J. of Chem. Phys. 21, $1087-1092$.

Mosegaard, K. (1998). Resolution analysis of general inverse problems through inverse Monte Carlo sampling. Inverse Problems 14, 405-426.

Mosegaard, K. and Tarantola, A. (1995). Monte Carlo sampling of solutions to inverse problems. Journal of Geophysical Research B 100, 12431-12447.

Møller, I., Jacobsen, B. H. and Christensen, N. B. (2001). Rapid inversion of 2-D geoelectrical data by multichannel devonvolution. Geophysics 66, $800-808$.

Nicholls, G. K. (1998). Bayesian image analysis with Markov chain Monte Carlo and coloured continuum triangulation models. Journal of the Royal Statistical Society Series B 60, 643-659.

Nielsen, O.B. (1973). Eocæne aflejringer i Ølst-området og deres indpasning i områdets kvartærgeologi (in Danish). Dansk Geologisk Forening, Årskrift 1972 , 100 - 110.

Panissod, C., Dabas, M., Hesse, A., Jolivet, A., Tabbagh, J. and Tabbagh, A. (1998). Recent developments in shallow-depth electrical and electrostatic prospecting using mobile arrays. Geophysics 63, $1542-1550$.

Pearl, J. (1988). Probabilistic Reasoning in Intelligent Systems: Networks of Plausible Inference. Morgan Kaufmann, San Mateo, California.

Robert, C. P. and Casella, G. (1999). Monte Carlo Statistical Methods. Springer-Verlag, New York.

Sørensen, K. I. (1996). Pulled array continuous electrical profiling. First Break 14, 85 - 90. 